\title{
Oral treatment with Euterpe oleracea Mart. (açaí) extract improves cardiac dysfunction and exercise intolerance in rats subjected to myocardial infarction
}

\author{
Gisele Zapata-Sudo ${ }^{1 *}$, Jaqueline S da Silva ${ }^{1 \dagger}$, Sharlene L Pereira ${ }^{1 \dagger}$, Pergentino JC Souza ${ }^{2 \dagger}$,
}

Roberto S de Moura ${ }^{2+}$ and Roberto Takashi Sudo ${ }^{1+}$

\begin{abstract}
Background: This study was designed to evaluate the cardioprotective effects of Euterpe oleracea Mart., popularly known as "açaí", on rats subjected to myocardial infarction (Ml).

Methods: Hydroalcoholic extracts of açaí were obtained from a decoction of the seeds. Two male Wistar rat groups were delineated: 1 ) the sham-operated group (control, $n=6$ ), with no surgical amendment, and 2 ) the Ml group ( $n=12)$, in which the anterior descendent coronary artery was occluded during surgery. Ml group was divided into two subgroups, in which rats were either treated with hydroalcoholic extract of Euterpe oleracea seeds (100 mg/kg/day p.o.) or received no treatment. Treatment began on the day of surgery, and lasted 4 weeks. Subsequently, rats were subject to an exercise test protocol, hemodynamic evaluation, and histological analysis of the left ventricle. Groups were compared using one-way analysis of variance (ANOVA), followed by Dunnett's test.

Results: The total running distance of sham rats was $1339.0 \pm 276.6 \mathrm{~m}$, Ml rats was $177.6 \pm 15.8 \mathrm{~m}(P<0.05)$, and Ml-açaí rats was $969.9 \pm 362.2 \mathrm{~m}$. Systolic arterial pressure was significantly decreased in Ml rats $(86.88 \pm 4.62 \mathrm{mmHg}$ ) compared to sham rats $(115.30 \pm 7.24 \mathrm{mmHg} ; P<0.05)$. Açaí treatment prevented a reduction in systolic arterial pressure $(130.00 \pm$ $8.16 \mathrm{mmHg}$ ) compared to $\mathrm{Ml}$ rats $(P<0.05)$. Left ventricular (LV) end-diastolic pressure was significantly augmented in $\mathrm{Ml}$ rats $(17.62 \pm 1.21 \mathrm{mmHg})$ compared to sham rats $(4.15 \pm 1.60 \mathrm{mmHg} ; P<0.05)$, but was $3.69 \pm 2.69 \mathrm{mmHg}$ in açaí-treated rats $(P<0.05$ vs. MI). The LV relaxation rate $(-\mathrm{dp} / \mathrm{dt})$ was reduced in $\mathrm{Ml}$ rats compared to the sham group, whereas açaí treatment prevented this reduction. Açaí treatment prevented cardiac hypertrophy and LV fibrosis in MI rats.
\end{abstract}

Conclusions: Euterpe oleracea treatment of MI rats prevented the development of exercise intolerance, cardiac hypertrophy, fibrosis, and dysfunction.

Keywords: Euterpe oleracea, Myocardial infarction, Exercise intolerance, Left ventricular dysfunction, Cardiac hypertrophy

\section{Background}

Myocardial infarction (MI) is a major public health problem and the leading cause of human mortality in both developed and developing countries. MI is an acute condition of myocardial necrosis, which is caused by an imbalance between the coronary blood supply

\footnotetext{
* Correspondence: gsudo@icb.ufrj.br

${ }^{\dagger}$ Equal contributors

'Programa de Desenvolvimento de Fármacos, Instituto de Ciências

Biomédicas, Universidade Federal do Rio de Janeiro, Rio de Janeiro, RJ, Brazil Full list of author information is available at the end of the article
}

and myocardial demand. This condition is regularly followed by several biochemical alterations to the body, including lipid peroxidation, hyperlipidemia, free radical damage, and hyperglycemia, leading to qualitative and quantitative changes to the myocardium [1]. Treatment of ischemic injury includes restoration of the blood supply to ischemic tissue and prevention of damage inflicted at the time of injury.

Reactive oxygen species (ROS) play an important role in oxidative stress and related myocardial damage. Increasing numbers of ROS-like hydroxyl radicals and superoxide

\section{Ciomed Central}


anions during heart ischemia lead to destruction of the cell membrane, development of lipid peroxides, and damage to the antioxidative defense system $[2,3]$. Experimental and clinical studies have shown that the infarct size of myocardial necrosis may be limited by the development of endogenous antioxidant enzymes and the suppression of free radical generation [4].

Post-MI myocardial damage is attenuated by antiinflammatory, anti-fibrotic, and vasodilator agents. MI causes inflammation and leukocyte recruitment to the injured myocardium and renal glomerulus [5]. After MI, myofibroblast persistence may contribute to fibrosis and adverse myocardial remodeling, particularly if the myofibroblasts remain active in healthy areas of the heart that are located at a distance from the original site of injury (reactive fibrosis). Fibrosis in the remote myocardium inevitably leads to increased myocardial stiffness, resulting in systolic and diastolic dysfunction, left ventricular (LV) hypertrophy, arrhythmia, neurohormonal activation, and, ultimately, heart failure [6].

Recently, there has been growing interest toward establishing the therapeutic potential of natural products against several diseases. For instance, the consumption of plant items, such as antioxidant supplements or antioxidantcontaining foods, might be used to protect against various diseases, including cardiovascular diseases [7]. Euterpe oleracea Mart., popularly known as "açaí," is widely cultivated in the Amazon region of Brazil. Chemical studies have shown that this purple fruit contains hydroxybenzoic acids, antioxidant polyphenolics, flavan-3-ols, and anthocyanins, predominantly cyanidin 3-O-rutinoside and cyanidin 3-Oglucuronide [8-10]. Açaí exhibits anti-inflammatory action through the inhibition of cyclooxygenases 1 and 2 [11], vasodilator effect [12], inhibition of nitric oxide (NO) production, as well as inducible nitric oxide synthase (iNOS) activity and expression [13], and antioxidant properties in acute lung inflammation [14].

Although E. oleracea is an important medicinal plant that has antioxidant, anti-inflammatory, and vasodilator properties, its cardioprotective activity against MI has not been studied. Therefore, in this study, we investigated the effects of the oral administration of hydroalcoholic extract from the seeds of E. oleracea fruit on cardiac dysfunction and exercise intolerance of rats subjected to MI.

\section{Methods}

\section{Preparation of extract from Euterpe oleracea (açaí)}

E. oleracea Mart. fruit was obtained from Amazon Bay (Belém do Pará, Pará, Brazil) excicata number 29052 Museu Goeldi - Belem do Para. Hydroalcoholic extracts were obtained from the seed (stone), as previously described [14]. In brief, approximately $200 \mathrm{~g}$ of açaí stone were boiled in $400 \mathrm{ml}$ of water for $5 \mathrm{~min}$, mixed for $2 \mathrm{~min}$, and then boiled again for $5 \mathrm{~min}$. The decoction was cooled to room temperature and extracted by adding $400 \mathrm{ml}$ of ethanol, with shaking for $2 \mathrm{~h}$. The extract was stored in dark bottles at $4^{\circ} \mathrm{C}$ for 10 days.

After this maceration period, the açaí hydroalcoholic extracts were filtered through \#1 Whatman filter paper. The ethanol was evaporated using a rotary evaporator (Fisatom Equipamentos Científicos Ltda São Paulo, São Paulo, Brazil) under low pressure at $55^{\circ} \mathrm{C}$. The extract was lyophilized (LIOTOP model 202, Fisatom Equipamentos Científicos Ltda São Paulo, São Paulo, Brazil) at temperatures ranging from -30 to $-40^{\circ} \mathrm{C}$ under vacuum at $200 \mathrm{mmHg}$. The extract was frozen at $-20^{\circ} \mathrm{C}$ until use. Typically $100 \mathrm{~g}$ of stone yielded approximately $5 \mathrm{~g}$ of lyophilized extract. Highperformance liquid chromatography (HPLC) analysis was performed as described previously [14].

\section{Animals}

This study was performed on male Wistar rats (body weight: 150-200 g). Experimental protocols were approved by the Animal Care and Use Committee of the Federal University of Rio de Janeiro, Brazil.

\section{Coronary artery ligation}

Under sevoflurane inhalation anesthesia (3\%), a left thoracotomy was performed in the fourth or fifth intercostal space. While the heart was exteriorized, the anterior descendent coronary artery was ligated by 1-2 mm from its origin with a 6.0 suture [15]. In the sham group, the same procedure was employed, but the suture was not tied. Rats were divided into sham-operated and MI groups (6 rats per group), which either received treatment with E. oleracea seed hydroalcoholic extract (açaí, $100 \mathrm{mg} / \mathrm{kg} /$ day p.o. by gavage) or not. Treatment with açaí began on the day of surgery and lasted 4 weeks. The açaí hydroalcoholic extract was dissolved in water.

\section{Exercise test protocol}

Four weeks after the surgery, rats performed a graded treadmill run to fatigue on a customized rodent treadmill (EP-131, Insight, São Paulo, Brazil). The protocol involved the rats running in three steps, with a progressive increase in treadmill speed: 1) $8 \mathrm{~m} \mathrm{~min}^{-1}$, 2) $12 \mathrm{~m} \mathrm{~min}^{-1}$, and 3) $18 \mathrm{~m} \mathrm{~min}^{-1}$. The two initial steps lasted for $3 \mathrm{~min}$ each, whereas rats continued running in the final step until they reached the point of fatigue, which was confirmed by the loss of the animal righting reflex. Subsequently, the total running distance was obtained.

\section{Cardiac mass and hemodynamic measurements}

Four weeks after surgery, the animals were anesthetized with pentobarbital sodium (50 mg/kg, i.p.), and prepared for arterial blood and LV pressure measurements. A catheter (PE-50) was introduced into the right carotid artery, and arterial blood pressure was measured by PowerLab 
(ADInstruments, Sydney, Australia) monitoring equipment. Subsequently, the catheter was introduced into the LV to record LV pressure. Hemodynamic values were automatically calculated using a physiological data acquisition system LabChart 7.0 (ADInstruments, Sydney, Australia). Anesthetized animals were euthanized at the end of the experiment. Afterwards, their hearts were weighed, and the heart weight to body mass ratio was calculated.

\section{Histological analysis}

The hearts of all rats from the experimental groups were harvested, instilled with $10 \%$ neutral buffered formalin, and immersed in the same fixative. Tissues were longitudinally cut, routinely processed, and embedded in paraffin blocks. Sections (7- $\mu \mathrm{m}$-thick) were stained with picrosirius red and examined by optical microscopy. The volume fraction of collagen (\%) was determined by measuring the area of stained LV within a given field. The stained area was calculated as a percentage of the total area within a field (Image-Pro Plus). Six fields were analyzed from each tissue and averaged.

\section{Statistical analysis}

All data were expressed as the mean \pm standard error of the mean (SEM). Differences among groups were considered statistically significant when the $P$ value was $<0.05$ using one-way analysis of variance (ANOVA), followed by a post-hoc Dunnett's test.

\section{Results}

\section{Reduced exercise intolerance in MI rats with açaí treatment}

Four weeks after surgery, the animals were submitted to a treadmill test. The total running distance for sham rats was $1339.0 \pm 276.6 \mathrm{~m}$, compared to $177.6 \pm 15.8 \mathrm{~m}$ for $\mathrm{MI}$ rats $(P<0.05$ vs. sham $)$ and $969.9 \pm 362.2 \mathrm{~m}$ for MI-açaí rats $(P<0.05$ vs. MI; Figure 1$)$.

\section{Açaí administration normalizes $\mathrm{MI}$ rat hemodynamic and cardiac hypertrophy}

After oral treatment with açaí extract, rats were subject to hemodynamic analysis and cardiac hypertrophy evaluation. Systolic arterial pressure was decreased in MI rats (86.88 \pm $4.62 \mathrm{mmHg})$ compared to sham rats $(115.30 \pm 7.24 \mathrm{mmHg}$; $P<0.05$; Figure 2A). Açaí treatment prevented the reduction of systolic arterial pressure $(130.00 \pm 8.16 \mathrm{mmHg})$ compared to $\mathrm{MI}$ rats $(P<0.05$ vs. $\mathrm{MI}$; Figure $2 \mathrm{~A})$. The hemodynamic data showed no significant difference in the diastolic arterial pressure of the studied groups (Figure 2B).

The LV systolic pressure of MI rats $(97.29 \pm 6.63 \mathrm{mmHg})$ was decreased compared to sham rats $(124.20 \pm 5.88 \mathrm{mmHg}$; $P<0.05)$ and açaí-treated rats $(148.60 \pm 8.47 \mathrm{mmHg} ; P<0.05$ vs. sham and MI; Figure 3A). The LV end-diastolic pressure was augmented in MI rats $(17.62 \pm 1.21 \mathrm{mmHg})$

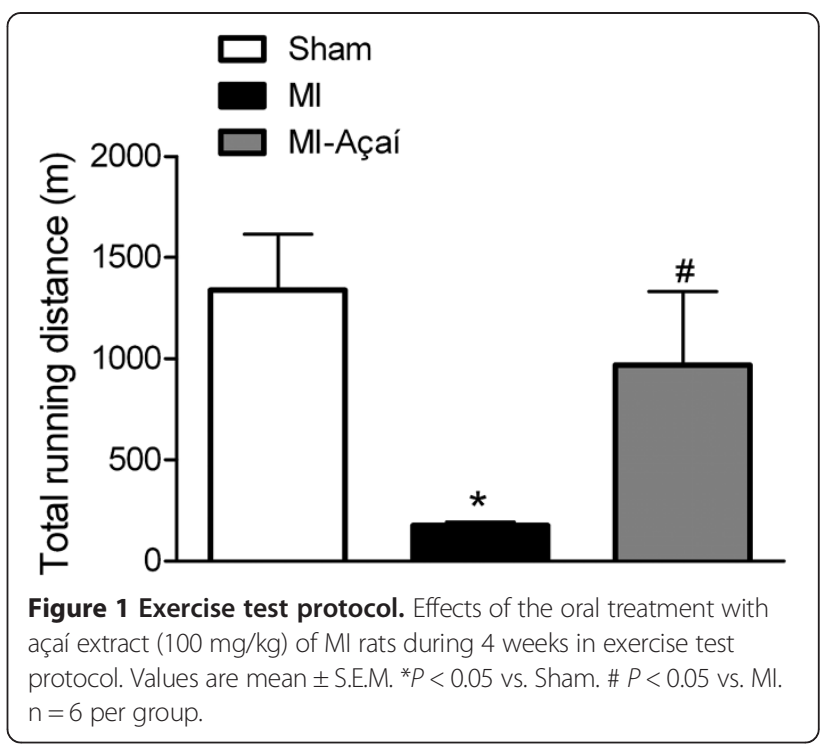

compared to sham rats $(4.15 \pm 1.60 \mathrm{mmHg} P<0.05)$, and it was $3.69 \pm 2.69 \mathrm{mmHg}$ in MI-açaí rats $(P<0.05$ vs. MI; Figure $3 \mathrm{~B})$. The LV relaxation rate (assessed by $-\mathrm{dp} / \mathrm{dt}$ ) was reduced in MI rats compared to sham rats, whereas treatment with açaí prevented this reduction (Figure 3C). We did not observe any significant differences in LV contractility (assessed by $+\mathrm{dp} / \mathrm{dt}$ ) among the study groups (Figure 3D).

The heart weight to body weight ratio was significantly greater in MI rats $(7.07 \pm 0.55 \mathrm{mg} / \mathrm{g})$ compared to sham rats $(4.89 \pm 0.25 \mathrm{mg} / \mathrm{g} ; P<0.05)$. Açaí treatment prevented cardiac hypertrophy, with a heart weight to body weight ratio of $5.17 \pm 0.37 \mathrm{mg} / \mathrm{g}(P<0.05$ vs. MI; Figure $4 \mathrm{~A})$. There was no significant difference in rat body weight among the experimental groups (Figure 4B).

\section{Reduction of LV fibrosis in MI rats after açaí extract treatment}

After the oral treatment of MI rats with açaí extract, collagen deposition in the LV was determined by picrosirius red staining (Figure 5A). The volume fraction of collagen (\%) was $10.39 \pm 1.06$ in sham rats compared to $52.39 \pm$ 3.85 in MI rats $(P<0.05)$. Oral treatment with açaí extract prevented LV fibrosis, with a collagen volume fraction (\%) of $23.57 \pm 6.78(P<0.05$ vs. MI; Figure $5 \mathrm{~B})$.

\section{Discussion}

In the present study, we demonstrated that the development of deleterious effects in MI rats was prevented by oral treatment with açaí extract for 4 weeks. Treatment with açaí prevented the reduction of exercise resistance during the exercise test procedure, in addition to preventing cardiac hypertrophy, LV fibrosis, and hemodynamic changes, such as reductions of systolic arterial pressure, 
A

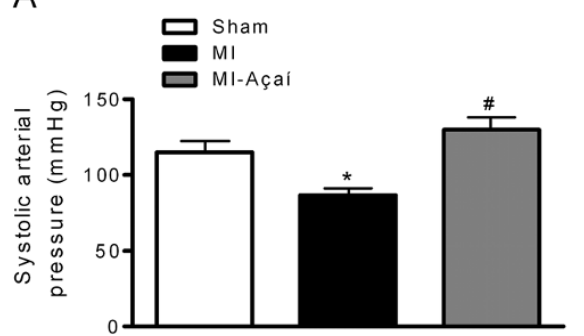

B

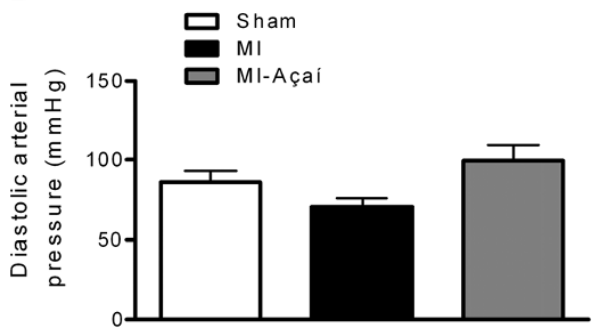

Figure 2 Systolic and diastolic arterial pressures. Effects of açaí extract (100 mg/kg) in systolic (A) and diastolic (B) arterial pressures of Ml rats. Values are mean \pm S.E.M. ${ }^{*} P<0.05$ vs. Sham. \# $P<0.05$ vs. MI. $n=6$ per group.

LV systolic pressure, and relaxation rate $(-\mathrm{dp} / \mathrm{dt})$, and enhancement of the LV end-diastolic pressure.

In the exercise test protocol, MI rats ran for shorter distances compared to sham rats. Intolerance to physical exercise has been characterized in different animal models of heart failure [16], as well as in humans [17-19]. In this study, açaí extract treatment of the MI group for 4 weeks prevented a reduction in exercise tolerance, with rats performing at a similar level to sham rats. This beneficial effect might be partly related to the vasodilator action of açaí through the NO/sGC/cGMP pathway [12]. This action might improve blood flow in the skeletal muscle and, hence, exercise capacity. In addition, diastolic dysfunction is the primary mechanism responsible for dyspnea and muscle fatigue in heart failure subjects [20]. The role of açaí in preventing exercise intolerance might also be related to the delayed development of cardiac hypertrophy, reduction in the LV end-diastolic pressure, and an increase in the rate of relaxation $(-\mathrm{dp} / \mathrm{dt})$ of cardiac muscle from MI rats.

Plasma levels of proinflammatory cytokines seem to increase with heart failure, and have a predictive prognostic value [21]. MI induces leukocyte recruitment to the injured myocardium, which contributes to myocardial damage [5] which could be reduced by açai extract. Several studies have demonstrated the anti-inflammatory action of E. oleracea. More recently, Moura et al. [14] demonstrated that E. oleracea extract reduces acute lung inflammation
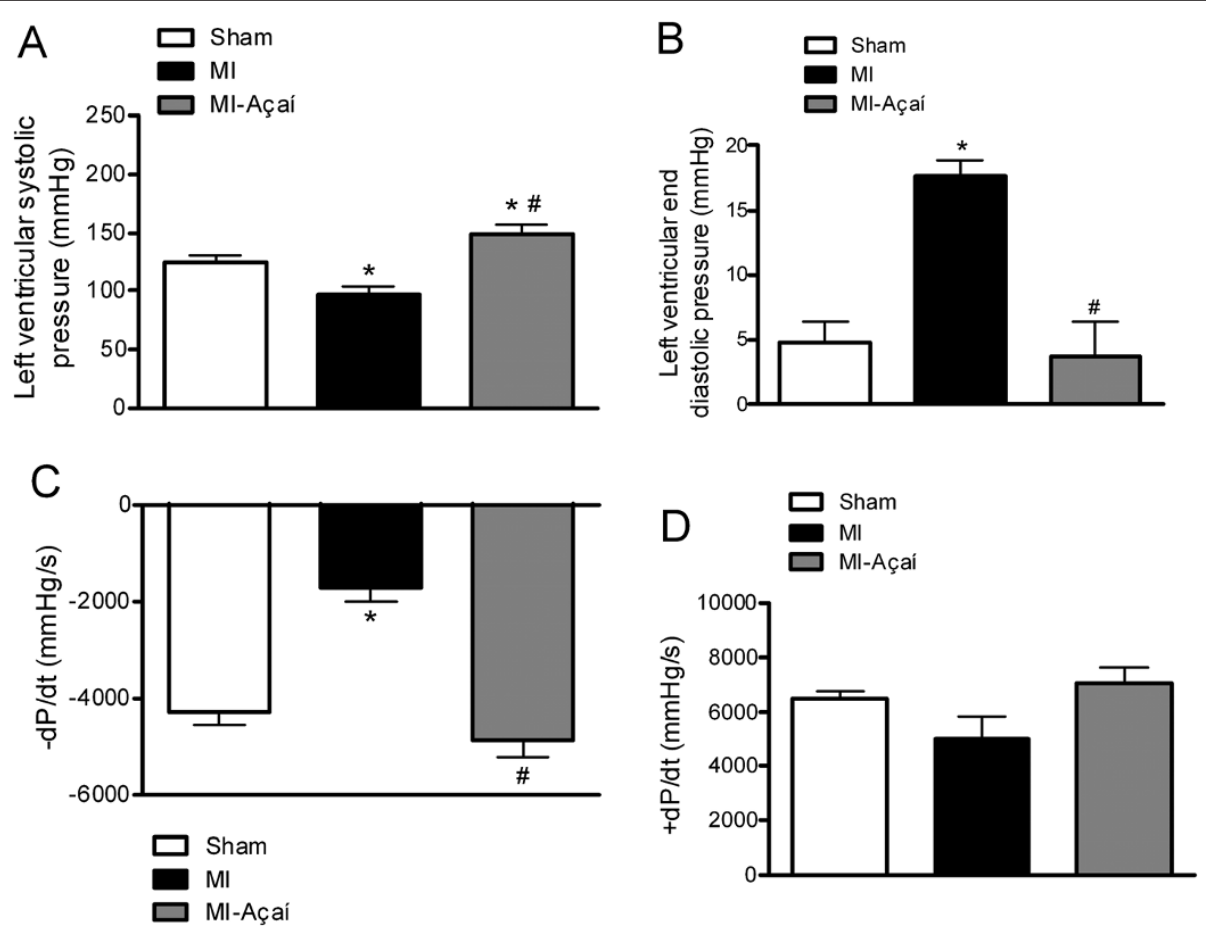

Figure 3 Hemodynamic data. Hemodynamic data of Sham, Ml and MI rats treated with açaí extract (100 mg/kg) during 4 weeks. The analyzed parameters were (A) left ventricular systolic pressure, (B) left ventricular end diastolic pressure, (C) $-d P / d t$ and (D) $+d P / d t$. Values are mean \pm S.E. M. ${ }^{*} P<0.05$ vs. Sham. \# $P<0.05$ vs. MI. $\mathrm{n}=6$ per group. 

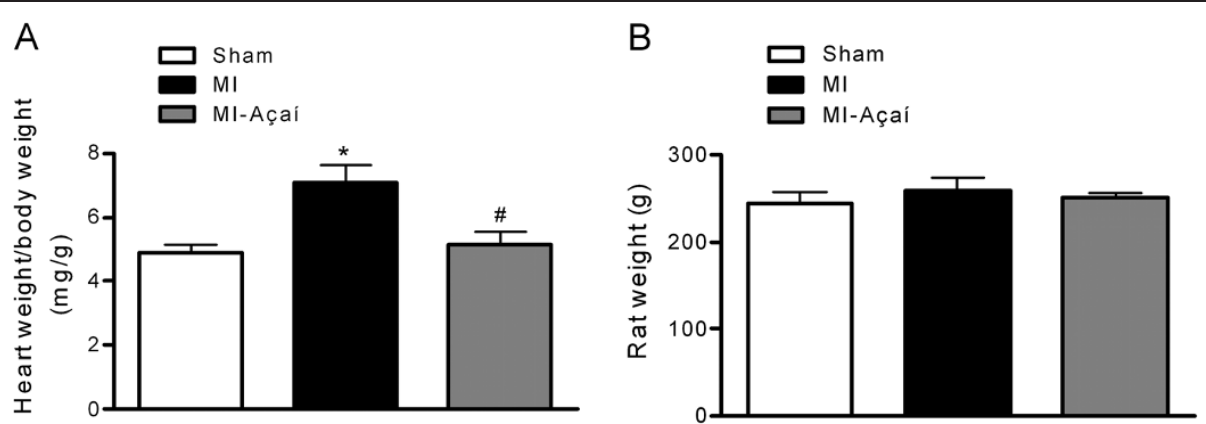

Figure 4 Cardiac hypertrophy. (A) Ratio of heart weight to body weight and (B) body weight of Sham, Ml and Ml rats treated with açaí extract $(100 \mathrm{mg} / \mathrm{kg})$ during 4 weeks. Values are mean \pm S.E.M. ${ }^{*} P<0.05$ vs. Sham. \# $P<0.05$ vs. Ml. $n=6$ per group.

in mice, by decreasing the numbers of alveolar macrophages and neutrophils in lung sections and decreasing TNF- $\alpha$ expression in lung homogenates. Another important action of E. oleracea in the controlling inflammatory process is the inhibition of NO production by reducing the expression of iNOS [13].

ROS and oxidative stress might also exacerbate myocardial damage after MI. Increasing numbers of hydroxyl radicals and superoxide anions during heart ischemia lead to destruction of the cell membrane, lipid peroxidation, and damage to the antioxidative defense system [2,3]. Experimental and clinical studies have shown that the infarct size of myocardial necrosis may be limited by antioxidant agents [4]. Several researchers have demonstrated the beneficial effects of açaí as an antioxidant agent [11,22-24]. Açaí juice increases the expression of antioxidant enzymes, such as glutathione reductase and glutathione peroxidase 3, in the aorta of apolipoprotein E-deficient mice.

Myofibroblast persistence after MI promotes fibrosis and myocardial remodeling, leading to increased myocardial

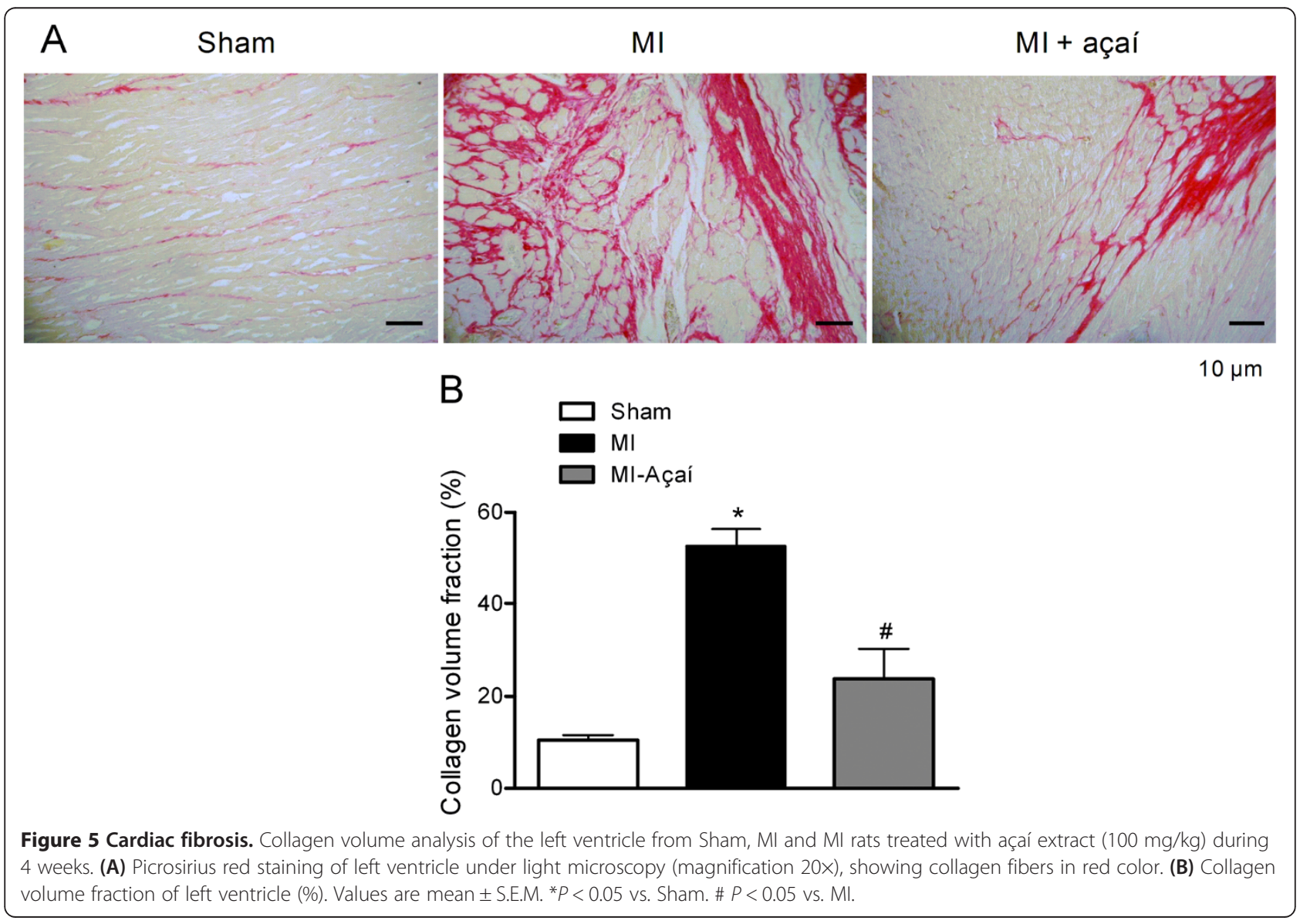


stiffness, systolic and diastolic dysfunction, LV hypertrophy, arrhythmia, neurohormonal activation, and, ultimately, heart failure [6]. In the current study, the development of cardiac fibrosis was prevented in MI rats that were treated orally with açaí extract, based on the observed reduction of collagen deposition in the LV. Thus, treatment with açaí extract has beneficial effects in delaying cardiac remodeling, and represents a novel therapeutic agent to prevent heart failure resulting from MI.

\section{Conclusions}

E. oleracea treatment for 4 weeks prevented the development of exercise intolerance, cardiac hypertrophy, fibrosis, and dysfunction in MI rats. These beneficial effects might be related to the antioxidant, vasodilator, and anti-inflammatory properties of its seed extract.

\section{Competing interests}

The authors declare that they have no competing interests.

\section{Authors' contributions}

RTS, GZS and RSM idealized the study, helped to draft the manuscript, supervised the study design and revised the manuscript. JSS and SLP performed the pharmacological experimental work and analyzed the data. RSM, PJCS carried out the preparation of extract from Euterpe oleracea. All authors read and approved the final manuscript.

\section{Acknowledgements}

This work was supported by Coordenação de Aperfeiçoamento de Pessoal de Nível Superior (CAPES), Fundação Universitária Jose Bonifácio (FUJB), Fundação Carlos Chagas Filho de Amparo à Pesquisa do Estado do Rio de Janeiro (FAPERJ), Conselho Nacional de Desenvolvimento Científico e Tecnológico (CNPq).

\section{Author details}

${ }^{1}$ Programa de Desenvolvimento de Fármacos, Instituto de Ciências Biomédicas, Universidade Federal do Rio de Janeiro, Rio de Janeiro, RJ, Brazil. ${ }^{2}$ Departamento de Farmacologia e Psicobiologia, Instituto de Biologia Roberto Alcântara Gomes, Universidade do Estado do Rio de Janeiro, Rio de Janeiro, RJ, Brazil.

Received: 7 October 2013 Accepted: 30 June 2014

Published: 8 July 2014

\section{References}

1. Kumar JS, Menon VP: Changes in levels of lipid peroxides and activity of superoxide dismutase and catalase in diabetes associated with myocardial infarction. Indian J Exp Biol 1992, 30:122-127.

2. De Biase L, Pinatelli P, Lenti L, Tocci G, Piccioni F, Riondino S: Enhanced TNF alpha and oxidative stress in patients with heart failure: effect of TNF alpha on platelet $\mathrm{O}_{2}$ - production. Thromb Haemost 2003, 90:317-325.

3. Rajadurai M, Prince PS: Preventive effect of naringin on cardiac markers, electrocardiographic patterns and lysosomal hydrolases in normal and isoproterenol-induced myocardial infarction in Wistar rats. Toxicology 2007, 230:178-188.

4. Nakamura $T$, Nishi $H$, Kokusenya $Y$, Hirota K, Miura Y: Mechanism of antioxidative activity of fluvastatin-determination of the active position. Chem Pharm Bull 2000, 48:235-237.

5. Ruparelia N, Digby JE, Jefferson A, Medway DJ, Neubauer S, Lygate CA Choudhury RP: Myocardial infarction causes inflammation and leukocyte recruitment at remote sites in the myocardium and in the renal glomerulus. Inflamn Res 2013, 62:515-525.

6. Turner NA, Porter KE: Function and fate of myofibroblasts after myocardial infarction. Fibrogenesis Tissue Repair 2013, 6(5):1-10.

7. Ozen T, Turkeku I: Antioxidant activities of Sarcodon imbricatum wildly grown in the black sea region of Turkey. Pharmacogn Mag 2010, 6:89-97.
8. Del Pozo-Insfran D, Brenes CH, Talcott ST: Phytochemical composition and pigment stability of Açaí (Euterpe oleracea Mart.). J Agric Food Chem 2004, 52:1539-1545.

9. Lichtenthaler R, Rodrigues RB, Maia JG, Papagiannopoulos M, Fabricius H, Marx F: Total oxidant scavenging capacities of Euterpe oleracea Mart. (Açaí) fruits. Int J Food Sci Nutr 2005, 56:53-64.

10. Rodrigues RB, Lichtenthaler R, Zimmermann BF, Papagiannopoulos M, Fabricius H, Marx F, Maia JG, Almeida O: Total oxidant scavenging capacity of Euterpe oleracea Mart. (açaí) seeds and identification of their polyphenolic compounds. J Agric Food Chem 2006, 54:4162-4167.

11. Schauss AG, Wu X, Prior RL, Ou B, Huang D, Owens J, Agarwal A, Jensen GS, Hart AN, Shanbrom E: Antioxidant capacity and other bioactivities of the freeze-dried Amazonian palm berry, Euterpe oleraceae mart. (AÇAí). J Agric Food Chem 2006, 54:8604-8610.

12. Rocha AP, Carvalho LC, Sousa MA, Madeira SV, Sousa PJ, Tano T, SchiniKerth VB, Resende AC, de Moura RS: Endothelium-dependent vasodilator effect of Euterpe oleracea Mart. (Açaí) extracts in mesenteric vascular bed of the rat. Vascul Pharmacol 2007, 46:97-104.

13. Matheus ME, de Oliveira SBF, Silveira CS, Rodrigues VP, de Sousa FM, Fernandes PD: Inhibitory effects of Euterpe oleracea Mart. on nitric oxide production and iNOS expression. J Ethnopharmacol 2006, 107:291-296.

14. Moura RS, Ferreira TS, Lopes AA, Pires KM, Nesi RT, Resende AC, Souza PJ, Silva AJ, Borges RM, Porto LC, Valenca SS: Effects of Euterpe oleracea Mart. (AÇAí) extract in acute lung inflammation induced by cigarette smoke in the mouse. Phytomedicine 2012, 19(3-4):262-269.

15. Gabriel D, Silva JS, Kummerle AE, Sudo RT, Landgraf SS, Caruso-Neves C, Fraga CAM, Barreiro EJ, Zapata-Sudo G: LASSBio-294, a compound with inotropic and lusitropic activity, decreases cardiac remodeling and improves $\mathrm{Ca} 2+$ influx into sarcoplasmic reticulum after myocardial infarction. Am J Hypertens 2010, 23:1220-1227.

16. Pfeifer C, Mesch TI, Mcallister RM: Skeletal muscle oxidative capacity and exercise tolerance in rats with heart failure. Med Sci Sport Exerc 2001 33(4):542-548.

17. Sullivan MJ, Green HJ, Cobb FR: Altered skeletal muscle metabolic response to exercise in chronic heart failure. Relation to skeletal muscle aerobic enzyme activity. Circulation 1991, 84:1597-1607.

18. Abudiab MM, Redfield MM, Melenovsky V, Olson TP, Kass DA, Johnson BD, Borlaug BA: Cardiac output response to exercise in relation to metabolic demand in heart failure with preserved ejection fraction. Eur J Heart Fail 2013, 15(7):776-785.

19. Okita K, Kinugawa S, Tsutsui $\mathrm{H}$ : Exercise intolerance in chronic heart failure skeletal muscle dysfunction and potential therapies. Circ J 2013, 77:293-300.

20. Paulus WJ, Tschope C, Sanderson EJ, Rusconi C, Flachskampf FA Rademakers FE, Marino P, Smiseth OA, Keulenaer GD, Leite-Moreira AF, Borbely A, Edes I, Handoko ML, Heymans S, Pezzali N, Pieske B, Dickstein K, Fraser AG, Brutsaert DL: How to diagnose diastolic heart failure: a consensus statement on the diagnosis of heart failure with normal left ventricular ejection fraction by the heart failure and echocardiography associations of the European society of cardiology. Eur Heart J 2007, 28(20):2539-2550.

21. Mallat Z, Heymes C, Corbaz A, Logeart D, Alouani S, Cohen-Solal A: Evidence for altered interleukin 18 (IL)-18 pathway in human heart failure. FASEB J 2004, 18:1752-1754

22. Chin YW, Chai HB, Keller WJ, Kinghorn AD: Lignans and other constituents of the fruits of Euterpe oleracea (Açaí) with antioxidant and cytoprotective activities. J Agric Food Chem 2008, 56(17):7759-7764.

23. Pacheco-Palencia LA, Mertens-Talcott S, Talcott ST: Chemical composition, antioxidant properties, and thermal stability of a phytochemical enriched oil from Açaí (Euterpe oleracea Mart.). J Agric Food Chem 2008, 56(12):4631-4636.

24. Souza MO, Silva M, Silva ME, Oliveira Rde P, Pedrosa ML: Diet supplementation with açaí (Euterpe oleracea Mart.) pulp improves biomarkers of oxidative stress and the serum lipid profile in rats. Nutrition 2010, 26(7-8):804-810.

doi:10.1186/1472-6882-14-227

Cite this article as: Zapata-Sudo et al:: Oral treatment with Euterpe oleracea Mart. (açaí) extract improves cardiac dysfunction and exercise intolerance in rats subjected to myocardial infarction. BMC

Complementary and Alternative Medicine 2014 14:227. 